\title{
Supersonic jet formation and propagation in x-pinches
}

\author{
D.M. Haas · S.C. Bott · J. Kim • D.A. Mariscal · R.E. Madden · Y. Eshaq · U. Ueda • \\ G. Collins IV $\cdot$ K. Gunasekera $\cdot$ F.N. Beg $\cdot$ J.P. Chittenden $\cdot$ N. Niasse $\cdot$ C.A. Jennings
}

Received: 18 May 2010 / Accepted: 6 January 2011 / Published online: 19 January 2011

(C) The Author(s) 2011. This article is published with open access at Springerlink.com

\begin{abstract}
Observations of supersonic jet propagation in low-current $\mathrm{x}$-pinches are reported. X-pinches comprising of four $7.5 \mu \mathrm{m}$ diameter tungsten wires were driven by an $80 \mathrm{kA}, 50 \mathrm{~ns}$ current pulse from a compact pulser. Coronal plasma surrounding the wire cores was accelerated perpendicular to their surface due to the global $\mathbf{J} \times \mathbf{B}$ force, and traveled toward the axis of the $\mathrm{X}$-pinch to form an axially propagating jet. These jets moved towards the electrodes and, late in time $(\sim 150 \mathrm{~ns})$, were observed to propagate well above the anode with a velocity of $3.3 \pm 0.6 \times 10^{4} \mathrm{~m} / \mathrm{s}$. Tungsten jets remained collimated at distances of up to $16 \mathrm{~mm}$ from the cross point, and an estimate of the local sound speed gives a Mach number of $\sim 6$. This is the first demonstration that supersonic plasma jets can be produced using x-pinches with such a small, low current pulser. Experimental data compares well to three-dimensional simulations using the GORGON resistive MHD code, and possible scaling to astrophysical jets is discussed.
\end{abstract}

Keywords X-pinch · Jets · Supersonic outflows

\section{Introduction}

X-pinch plasmas, as described by Zakharov et al. (1982), have received a great deal of interest due to their de-

D.M. Haas · S.C. Bott $(\bowtie) \cdot$ J. Kim · D.A. Mariscal .

R.E. Madden · Y. Eshaq · U. Ueda · G. Collins IV ·

K. Gunasekera · F.N. Beg

University of California San Diego, La Jolla, CA, USA

e-mail: sbott@ucsd.edu

J.P. Chittenden - N. Niasse

Imperial College London, London, UK

C.A. Jennings

Sandia National Laboratories, Albuquerque, NM, USA velopment of exotic properties: up to $T_{\mathrm{e}} \sim 1 \mathrm{keV}$ and $n_{\mathrm{e}} \sim 10^{22} \mathrm{~cm}^{-3}$, in a highly localized hotspot (Zakharov et al. 1982; Shelkovenko et al. 2001a, 2001b, 2002). This has led to their application as an x-ray (1-10 keV) backlighter by Pikuz et al. (1997) and Shelkovenko et al. (2001b) for diagnosing dense plasmas. Pikuz et al. (2002) showed that these systems can be driven with currents as low as a few tens of kilo-amps, and are therefore a convenient and low-cost medium for the study of high energy density $\left(>10^{11} \mathrm{~J} / \mathrm{m}^{3}\right)$ and radiation dominated plasmas. While previous research has focused on the formation of the hot-spot, more recently studies have also investigated the low density coronal plasma ablated from the wires by the drive current.

In an X-pinch discharge, a large current is pulsed through two or more wires which are arranged in the form of an "X" between the electrodes (see Fig. 1). At the cross-point the current in each of the legs combines to compress the plasma through the self generated magnetic field producing a highly localized, hot, and dense region. At the same time, plasma is ablated along the length of the wires and is driven toward the axis by the global $\mathbf{J} \times \mathbf{B}$ force. The global Lorentz force varies with axial position, decreasing as the wire spacing increases away from the cross-point. This ablated plasma coalesces on the $\mathrm{x}$-pinch axis forming a jet-like structure, a process first described by Zakharov et al. (1982). More recently, Mitchell et al. (2000) studied the behavior of the coronal plasma on a $450 \mathrm{kA} \mathrm{x}$-pinch, and showed that the low-density coronal plasma contributes to jet formation through optical and XUV framing images. Beg et al. (2006) made similar observations with a $160 \mathrm{kA}$ generator, and additional investigations into the effects of wire coatings on the low density corona were reported by Haas et al. (2007). These studies focused on the plasma evolution between the electrodes where the wire ablation contributed to the zippered formation of the plasma structure. These 'jets' were 
Fig. 1 Schematic of the $x$-pinch (a) showing an enlarged view of the plasma flow convergence region (b). (c) shows anode setup indicating the placement of the wire limb blocks and the redefined convergence region. Finally (d) displays a series of areal electron density images showing the evolution of the plasma jet above the anode (a)

(b)

(c)

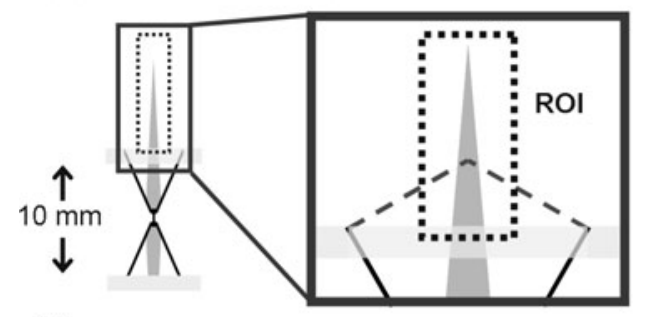

(d)

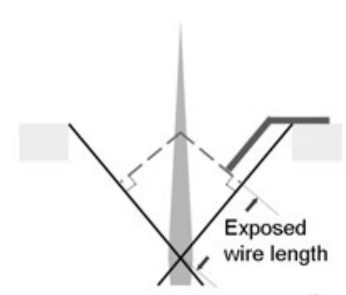

$\mathrm{n}_{\mathrm{e}, \text { areal }} \mathrm{cm}^{-2}$

$6 \times 10^{17}$

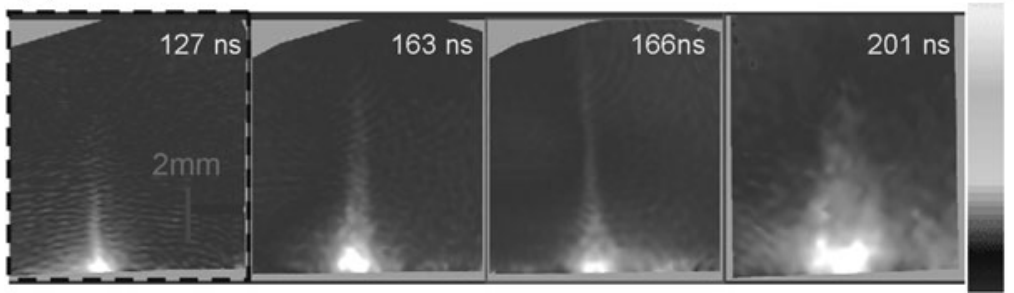

studied close to their formation region and hence were confined by the radial momentum of the ablated plasma flows.

Recently, there has been particular interest in laboratory produced supersonic plasma jets because of their potential similarities to astrophysical phenomena as investigated by Ryutov et al. (1999) and in further detail by Ryutov et al. (2000). To model such objects in the laboratory, Lebedev et al. (2002, 2004), and Ampleford et al. (2008) used conical wire arrays to produce highly collimated, radiative cooled jets. In this configuration, the ablated plasma from the wires is accelerated toward the axis via the global $\mathbf{J} \times \mathbf{B}$ force. This results in a flow, perpendicular to the wires, which converges to form a plasma jet at the axis. The combination of axial momentum from the ablated flow and a strong axial pressure gradient accelerates the jet above the electrode, while the radiative cooling resulted in a low divergence angle. Currently, these experiments are performed on 1 MA machines, which necessitate a laboratory footprint $>100 \mathrm{~m}^{2}$. In contrast, the $\mathrm{x}$-pinches discussed here are driven by a low current $(80 \mathrm{kA})$, compact $\left(<1 \mathrm{~m}^{2}\right)$ pulser. Producing astrophysically relevant plasma jets on such high repetition rate devices (up to 8 shots/day) opens the way for smaller scale experiments to contribute to the growing field of laboratory astrophysics. In this paper, we present the first evidence of the free-propagation of an $\mathrm{x}$-pinch jet above the anode far from the zipper region, using a compact pulsed power system.

\section{Experimental setup}

A conventional Marx-driven generator $(80 \mathrm{kA}$ with a rise time of $40 \mathrm{~ns}[10 \%-90 \%])$ was used to drive the $\mathrm{x}$-pinch load with an approximately $\sin ^{2}$ current waveform, for a complete description see Beg et al. (2006, 2007). Four
$7.5 \mu \mathrm{m}$ tungsten wires were used to construct the x-pinches. The wires were hung $12 \mathrm{~mm}$ apart between two electrodes which had a fixed separation of $1 \mathrm{~cm}$. One electrode was rotated $180^{\circ}$, relative to the other, causing the wires to make contact at a central point. The top electrode (anode) only contacted the wires at their widest separation and the central region was entirely open to allow uninhibited plasma flow and jet formation. When viewed radially, the upper plane of the anode is $7 \mathrm{~mm}$ above the cross point of the $\mathrm{x}$-pinch. The position of the jet studied can be seen in Fig. 1a, b, labeled region of interest, ROI (outlined by dashes). In an additional set of experiments, $300 \mu \mathrm{m}$ thick polypropylene shields of various lengths were installed on the anode for two of the four wires. The blocked wires were adjacent to one another and appeared on one 'side' of the x-pinch-see Fig. 1c. This prevented any plasma from the upper portion of the limbs from reaching the axis, and allowed an examination of the contribution of the low density flows (from the wires) far from the cross-point. In these experiments a portion of the anode was cut away allowing a diagnostic view of the plasma flow from the jet formation region to the $\mathrm{x}$-pinch cross point.

In order to monitor the electron density in the plasma, two interferometric images were captured during each experiment using a frequency doubled Nd-YAG laser $(\lambda=$ $532 \mathrm{~nm}$ ) with a pulse length of $5 \mathrm{~ns}$. The paths through the experiment were identical for the two imaging beams, which were temporally separated by $14 \mathrm{~ns}$. Upon exiting the chamber, the beams were split and propagated though a Nomarski (Benattar et al. 1979) and Mach-Zender interferometer independently. Images had a spatial resolution of $\sim 20 \mu \mathrm{m}$ and were recorded on CCD cameras. All image timings are given with respect to the start of the current pulse. The interferometer images were analyzed using the software package IDEA presented by Hipp et al. (2004). The regions of interest in the 
Fig. 2 Two areal density plots taken from the same shot (b).

The locations of the lineouts are indicated by vertical dashed lines. The graph in

(a) represents the respective areal density profiles as well as the average background density for each of the images. Linear extrapolations for the profiles are represented by dashed lines in respective colors. Vertical dashed lines mark the location of the end of the jet (a)

(b)

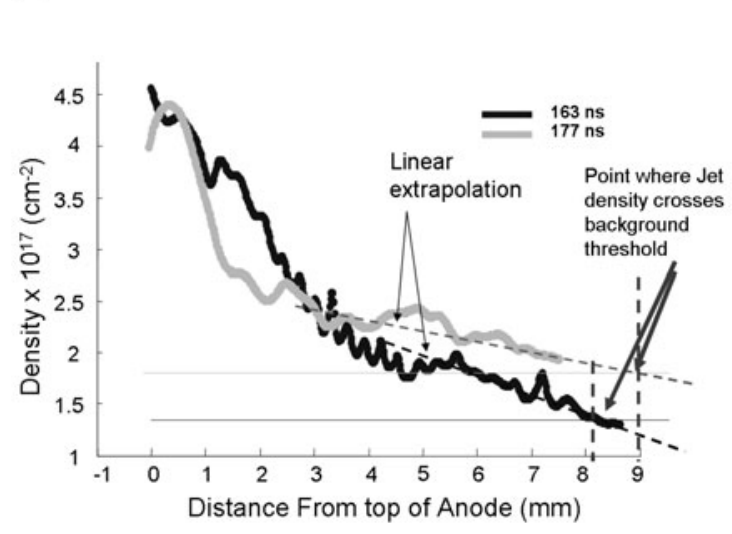

interferograms were masked and the Fast Fourier Transform (FFT) was performed to isolate the carrier frequency. Once complete, the inverse FFT was performed in order to extract the phase plot. The background phase was then removed in order to obtain the relative phase shift $(d \phi)$. This is related to the wavelength $(\lambda)$ and electron density $\left(n_{\mathrm{e}}\right)$ by the following relation:

$d \phi=\frac{\pi}{\lambda n_{\mathrm{c}}} \int_{-L / 2}^{L / 2} n_{\mathrm{e}} \cdot d l$,

where $L$ is the length scale of the plasma. Taking $\lambda$ to be $532 \mathrm{~nm}$, and dividing $d \phi$ by $2 \pi$ gives the relative fringe shift $f$. The areal electron density (in $\mathrm{cm}^{-2}$ ) is then:

$\int n_{\mathrm{e}}\left(\mathrm{cm}^{-3}\right) d l=4.2 \times 10^{17} f$.

Areal electron density maps appear in Fig. 1d. This sequence of images show densities of $\sim 5 \times 10^{17} \mathrm{~cm}^{-2}$ at the base of the jet, which increases with time, and $\sim 1 \times$ $10^{17} \mathrm{~cm}^{-2}$ at the tip of the jet (the determination of the jet tip position is discussed below). The error associated with the areal electron density is determined by the minimum observable fringe shift, which using this method is estimated as $\sim 1 / 10$ of the fringe spacing. Areal electron density measurements therefore have an error of $\sim 10 \%$ with a minimum detectable limit of $\sim 4 \times 10^{16} \mathrm{~cm}^{-2}$. The ablation of the plasma normal to the surface, and the angle the wires make with the axis of the pinch produce a plasma flow which converges above the anode plane (note that the electrical contact point of the wires is at the top of the anode). The maximum vertical extent of this convergence region, using the total extent of the wires in the $\mathrm{x}$-pinch, is $6 \mathrm{~mm}$ above the top of the anode, as indicated in Fig. $1 \mathrm{~b}$ by dashed lines. To be considered freely propagating, jets must be observed above this limit. In practice, the limit of convergent flow may be considerably lower than this, since the global magnetic field far from the cross-point is weak relative to the local field of the individual wires. Both the low density and small directed velocity of plasma from the extremes of the wires may contribute very little to the jet formation and confinement. This may result in a lower effective convergent flow limit, and hence a greater length of jet suitable for possible jet-target interaction experiments. The method of determining the jet tip position and an experimental estimation of the effective convergent flow limit are discussed in the following sections.

\section{Jet length determination and velocity}

The majority of the length of the jet has a clear contrast with the background density. However, the areal density at the tip of the jet provides only a low contrast, so a systematic method for determining the end of the jet was applied. A quantitative areal density profile was taken along the centerline of the jet, and these profiles were then plotted as a function of distance from the top of the anode. An average of the background areal electron density for each image was taken and plotted along with the density profile. The lengths of the jets were determined by the point at which jet density profile crossed the background threshold. This methodology was applied to a series of images, two of which can be seen in Fig. 2b. Note that both images in this figure were taken from the same shot. The location of the lineouts can be seen marked in grey, and the corresponding areal electron density profiles are plotted in Fig. 2a. For the image at 163 ns, the density profile clearly crosses the background limit threshold and gives a jet length of $8.1 \pm 0.05 \mathrm{~mm}$. However, for the image taken at 177 ns the jet has passed beyond our diagnostic view. We therefore used a linear extrapolation of the density profile to determine the length of the jet, which is shown as a dashed line in the plot. This fit will provide a 
minimum jet length and hence a lower limit on the jet velocity inferred from these measurements. The jet propagates a distance of $0.8 \pm 0.1 \mathrm{~mm}$ between 163 and $177 \mathrm{~ns}$.

Measurements of jet lengths from a series of experiments were plotted as a function of time, and are presented in Fig. 4a. Note that all jet lengths in this sequence are $>6 \mathrm{~mm}$ from the top of the anode; i.e. beyond the maximum limit of convergent flow discussed above. A linear fit of the data gives an average velocity of $3.3 \pm 0.6 \times 10^{4} \mathrm{~m} / \mathrm{s}$. The error associated with minimum detectable fringe shift is estimated at $\sim 10 \%$ as stated above. It is important to note here that these determinations inherently rely on a fixed ionization state (determined below) to locate the jet tip. If $Z$ changes along the jet as it cools, the velocity may be lower than suggested here. Although the low jet temperatures, and hence limited possible ionization states, suggest this effect will not change the final Mach number significantly.

\section{Effective limit to convergent flow}

To experimentally investigate the effective limit of the convergent flow, a portion of the 2 wires on one side of the $\mathrm{x}$ pinch were blocked using plastic shields. Ablated plasma flow from these regions was prevented from reaching the axis and contributing to the jet formation and confinement. Two shield lengths were used; one began $\sim 5.5 \mathrm{~mm}$ from the cross-point effectively reducing the wire to $65 \%$ of the original length, referred to as the 'short block'. The second began $\sim 3.5 \mathrm{~mm}$ from the cross point reducing the wire to $40 \%$ of the original length, and was labeled the 'long block'. In each case several experiments were performed; using the same methodology as above recorded jet images yielded areal electron density maps. These were then compared to measurements using the same set-up but without the shield in place. If the blocked plasma flow plays a significant role in the jet formation process, the jet trajectory should be deflected toward the block, since the kinetic pressure from the opposite side of the x-pinch will be greater. If the jet is not deflected beyond the typical range observed, the plasma flow prevented from reaching the axis does not contribute strongly to the jet formation. Consequently, this will set the point from which the effective limit of convergent flow can be determined. A comparison of the three cases at similar times is given in Fig. 3. The image appearing in (a) corresponds to an x-pinch in which all wires were unobstructed and served as a control case. The image in (b) was generated with an identical four wire pinch in which two wires were partially blocked by 'short' blocks, and (c) was recorded using two 'long blocks'. The radial profiles of the plasma jets are shown through the series of horizontal lineouts, taken at 1 millimeter axial increments, as indicated in each image.

In the unblocked and short block plots, a peak in the density profile is present near the axis $( \pm 0.15 \mathrm{~mm})$ for the entire a)
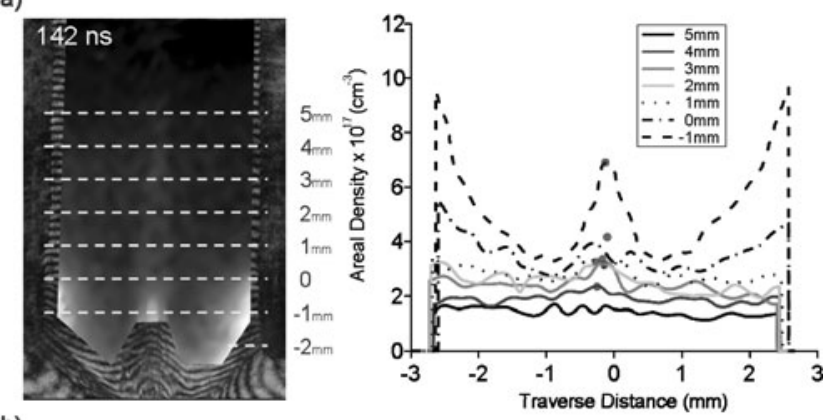

b)
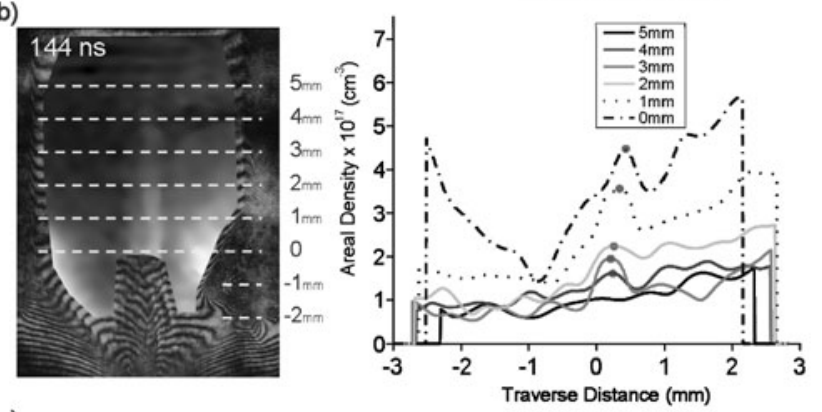

c)
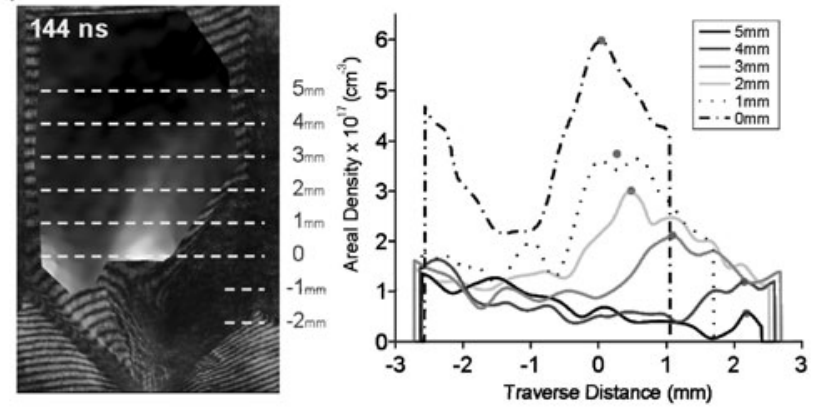

d)

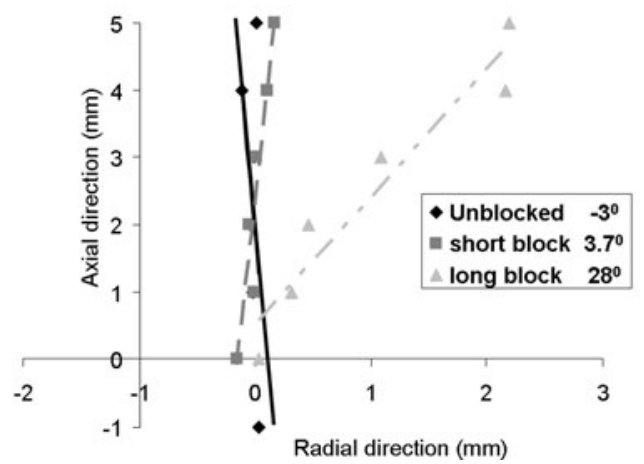

Fig. 3 Composite interferometric and areal density images, and lineouts from four wire, $7.5 \mu \mathrm{m}$ tungsten x-pinches showing (a) unblocked (b) 'short' block and (c) 'long' block cases. Lineouts are taken at the indicated axial positions. (d) Displays the radial location of the center of the jets vs. axial height for the unblocked, short, and long block cases

length of the jet. In both cases the jets appear to have a slight $\left(\sim 3^{\circ}\right)$ deviation from the axis. This is likely a result of the low symmetry of the flow reaching the axis, a result of the low wire number in these experiments, and is often evident (see Fig. 1). Due to similarities with the control case the 
Fig. 4 (a) Plot of jet length as a function of time (like markers indicate images from the same shot), a linear fit is included. In (c) two areal density images from the same shot with lineout positions indicated. (b) Plots the radial areal electron density profiles from the images in (c). The FWHM for the respective curves are indicated with arrows (a)

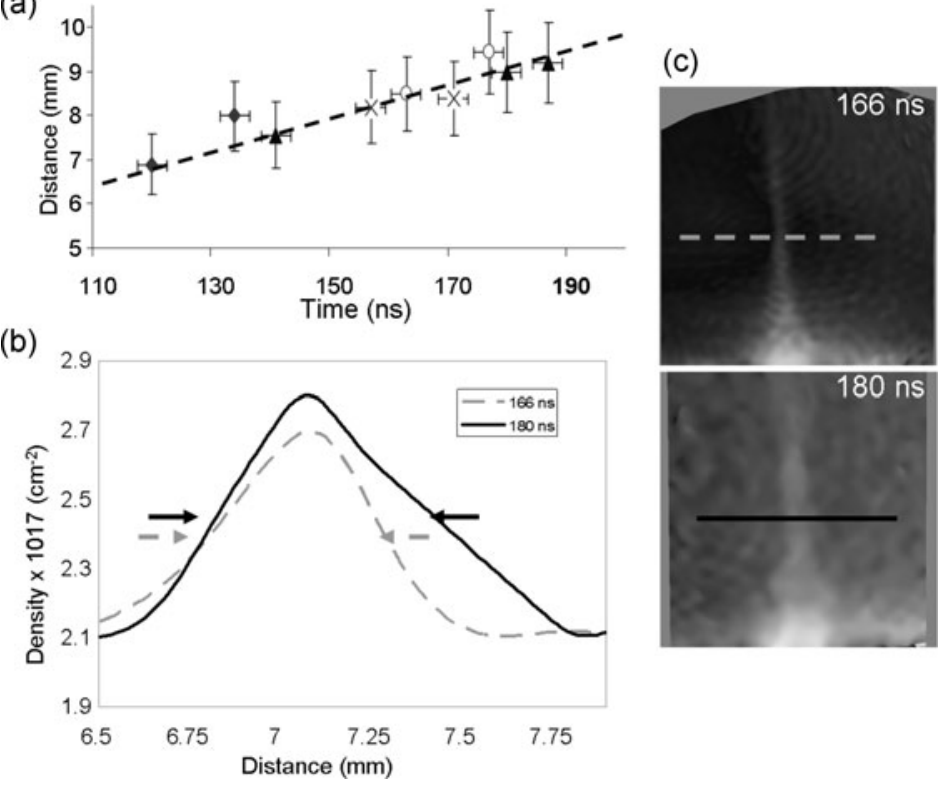

short blocks are ruled out as a source of the deviation. For the long block case, the peak of the density profile coincides with the experimental axis for the lineout closest to the cross point $(0 \mathrm{~mm})$. However, as the distance from the cross point increases, the peaks of the profiles migrate to the right. To more clearly show this, the peaks of the jet density profiles are graphed against their axial location in Fig. 3d. Linear fits highlight the angle of the jets which are $-3^{\circ}$ and $3.7^{\circ}$ for the unblocked and 'short' block cases respectively. Clearly divergent is the 'long' block case whose jet propagates to the right at an angle of $28^{\circ}$. Also evident is an increase in the density on the sides of the profiles. This is the expansion of the plastic shield under exposure to the XUV radiation from the wires themselves. In both blocked cases, this plasma is distinct from the jets and does not affect their trajectories.

From the above results, the short blocks obstructed plasma from the upper $2.8 \mathrm{~mm}$ ( $\sim 40 \%$ of the leg length) of the wires and did not significantly effect the propagation of the jets when compared to unblocked experiments. The 'long block' case, however, resulted in a significant change in the propagation of the axial plasma jet. In the latter case the jet deviated towards the blocked wires due to a lack of opposing momentum from these wires. These results demonstrate a clear difference in the contribution of the lower and upper portions of the x-pinch limbs. The inconsequential contributions from the upper $2.8 \mathrm{~mm}$ of the limbs prompted a reassessment of the convergence regions from the wire limbs. Retaining the assumption that the wire ablation occurs normal to the surface of the wires, the modified convergence region now ends $\sim 6.5 \mathrm{~mm}$ above the $\mathrm{x}$-pinch cross point. This is $6.5 \mathrm{~mm}$ lower than the initial maximum geometric limit. From the data in Fig. 1, the jets were recorded up to 19 jet radii above the convergent flow region.

\section{Estimation of dimensionless scaling parameters}

Beyond the convergent flow region the jets are no longer confined radially by the kinetic pressure ( $\left.\rho v_{\text {radial }}^{2}\right)$ of the ablated plasma flow, and hence will expand radially at the sound speed $\left(c_{\mathrm{S}}\right)$ while propagating axially. Through analysis of the jet evolution this value can be estimated. Figure $4 \mathrm{c}$ shows two images from the same shot. Areal electron density profiles were taken across the jet for both times, and the results were plotted in Fig. 4b. The FWHM of the jet diameter increases from $0.32 \mathrm{~mm}$ to $0.4 \mathrm{~mm}$ in $14 \mathrm{~ns}$. Averaging a number of shots analyzed in this way, an average sound speed of $c_{\mathrm{s}}=5.5 \pm 2.6 \times 10^{3} \mathrm{~m} / \mathrm{s}$ was obtained. To verify this value we can calculate the temperature and ionization state of the plasma and compare these with values expected from the experiment. Rearranging the sound speed equation gives:

$Z T_{\mathrm{e}}[\mathrm{eV}]=\left(\frac{\mathrm{c}_{\mathrm{s}}[\mathrm{cm} / \mathrm{s}]}{9.79 \times 10^{5}}\right)^{2} \frac{m_{\text {ion }}}{\gamma}$

For tungsten $\left(m_{\text {ion }}=184\right)$, and a $\gamma$ (ratio of specific heats) of 5/3, we find the value of $Z T_{\mathrm{e}}$ to be $35 \mathrm{eV}$. Using a coronal equilibrium ionization model appropriate for these densities, as described by Salzmann (1998), and assuming a $Z$ of $\sim 5$ gives an electron temperature of $7 \pm 1.6 \mathrm{eV}$. This value is consistent with the approximate temperature range inferred from time gated self-emission imaging of the jets using different sized pinholes as radiation filters. Filtration 
is due to the diffraction limited focusing for different energies. The values of the jet velocity and sound speed give a jet Mach number of $6 \pm 3$. Although the error on this measurement is large, within the limits the observed jets are supersonic.

The jets produced here may be of interest for laboratory astrophysics, and to determine their applicability several factors must be considered. A fluid description should be appropriate for the plasma, and restrictions on the Reynolds, and Peclet numbers ensure that the energy flow is dominated by heat convection, while dissipative effects such as viscosity, and thermal conductivity are negligible as concluded by Ryutov et al. (1999). For the jets to be collisional we require that the ion mean free path be less then the radius of the jets. Therefore the localization parameter must be:

$\delta_{\perp}=\frac{\lambda_{\text {mfp }}}{r_{\text {jet }}} \cong \frac{m_{\text {ion }}^{2} v_{\text {therm }}^{4}}{8 \pi Z^{4} e^{4} n_{\text {ion }} \ln \Lambda \sqrt{\pi / 2}} \frac{1}{r_{\text {jet }}} \ll 1$

Here the constant $e$ is in cgs units and the coulomb logarithm $\log (\Lambda)$ is $\sim 1$. Using the thermal velocity of tungsten ions at $11 \mathrm{eV}\left(v_{\text {therm }}=2 \times 10^{5} \mathrm{~cm} \mathrm{~s}^{-1}\right)$ and an ion density of $n_{\mathrm{i}}=2 \times 10^{18} \mathrm{~cm}^{-3}$, a localization parameter of $<10^{-3}$ is calculated. Substituting the values associated with the jets, both the Reynolds and Peclet numbers are $>10$. In addition to these limits, the three dimensionless parameters associated with radiative jets: the Mach number $M_{\mathrm{a}}$, cooling parameter $\chi$ (ratio of cooling length to jet radius), and density contrast ratio $\eta$ (ratio of jet density to background density), need to be quantified. For the jets discussed above $M_{\mathrm{a}} \sim 6$, and from self-emission imaging we estimate axial cooling lengths of $0.5-5 \mathrm{~mm}$ for jets with radii of $\sim 0.5 \mathrm{~mm}$, putting $\chi$ in the range of $0.1-1$. Finally, the jets in these experiments are propagating into vacuum, therefore $\eta \gg 1$.

\section{Comparison to 3D simulations}

We now compare the experimental results to simulations generated with the resistive MHD code GORGON. The MHD equations are solved on a three-dimensional grid in Cartesian geometry using a single fluid approximation. However, the ion and electron components of the plasma are treated independently and are allowed to vary from thermodynamic equilibrium with respect to one another and their relative energy equations are solved separately. An equation of state relates the pressure and internal energy, and the average ionization state of the plasma, $(Z)$ is calculated using an average-ion Thomas-Fermi model (Chittenden et al. 2004 and Chittenden and Jennings 2008). Radiation loss has been shown to be particularly importance to jet formation in wire arrays due to its contribution to radiative cooling, and is included through an optically thin line emission model. This
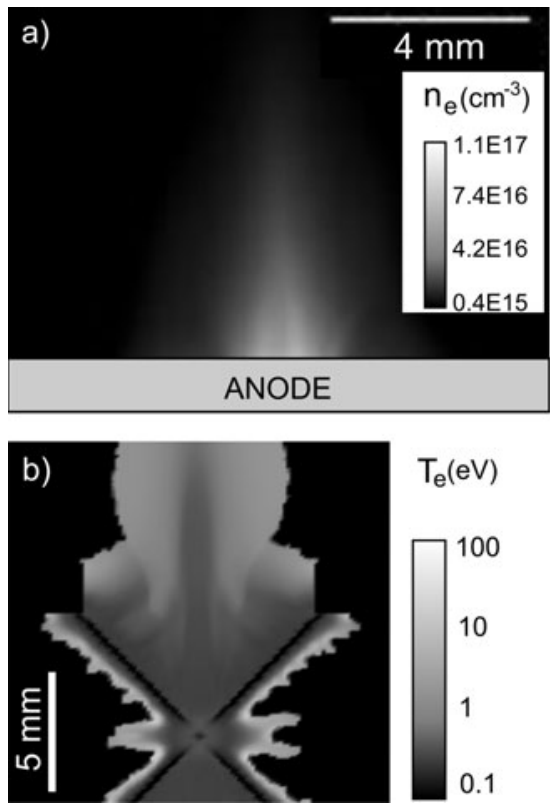

Fig. 5 (a) Areal electron density map of the jet at $140 \mathrm{~ns}$, and (b) a slice through the $\mathrm{x}$-pinch, electron temperature map $\left(T_{\mathrm{e}}\right.$ in $\left.\mathrm{eV}\right)$

allows an appropriate collapse of material on the axis. GORGON is currently used to model a wide range of wire array geometries, including imploding cylindrical arrays, conical jet-launching arrays, and radial array magnetic tower jets (Chittenden et al. 2004; Chittenden and Jennings 2008; Ampleford et al. 2007; Lebedev et al. 2005; Suzuki-Vidal et al. 2009).

The X-pinch jets presented above can be well represented by simulations, and information regarding the dynamics and plasma parameters can be obtained. The simulation is run on an $11 \times 11 \times 19.5 \mathrm{~mm}$ Cartesian grid with a $100 \mu \mathrm{m} \mathrm{cu}-$ bic cell size. The $\mathrm{x}$-pinch is located in the lower potion of the grid so that the region of interest, the area immediately above the anode, is included see Fig. 5. To model the above experimental results, the code was run with an $80 \mathrm{kA} 50 \mathrm{~ns}$ (risetime) current waveform to drive a four wire $\mathrm{x}$-pinch in which the wires were initially represented as a cold dense gas with a $T_{\mathrm{e}}=0.125 \mathrm{eV}$. Tungsten wires with the appropriate linear mass density are used; however, they initially occupy a single cell and are not resolved to the $7.5 \mu \mathrm{m}$ diameter (experiments). This set-up has been found to be sufficient to observe the dynamics of jet formation and propagation with no loss of information.

Many of the experimentally measured values are reproduced in the simulation, such as average jet velocity, diameter, and the expected temperature distribution. The areal electron density of the jet is obtained by integrating through the volumetric electron density in the $X Z$ plane (Fig. 5a). The electron density of the jet is found to be approximately an order of magnitude lower than observed in experiments, and the value of $n_{\mathrm{e}}=2 \times 10^{16} \mathrm{~cm}^{-2}$ is used to track the tip 
Fig. 6 (a) A portion of the velocity vector map (in $\mathrm{m} \mathrm{s}^{-1}$ ), at $t=140 \mathrm{~ns}$ after current initiation. (b) A portion of the magnetic field map, with magnitudes (in Tesla) at the same time
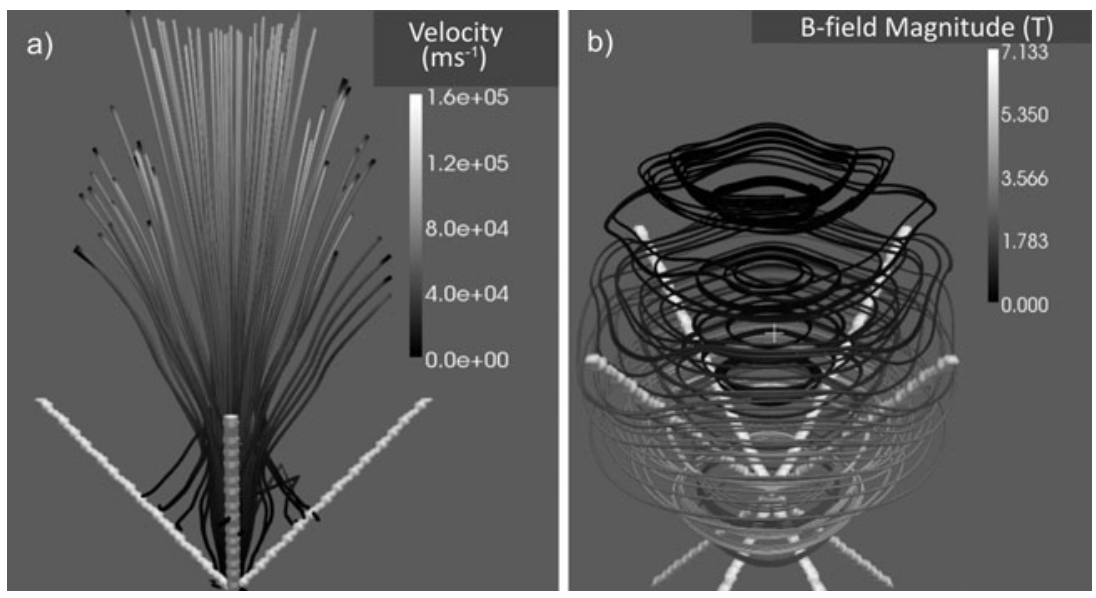

of the jet. Similar to experiment, the jet diameter (FWHM) is $\sim 2 \mathrm{~mm}$ at $140 \mathrm{~ns}$, as measured above the convergence regions and dissembles late in time ( $>180 \mathrm{~ns}$ ). Figure $6 \mathrm{a}$ shows relatively low velocity flow from the wires in contrast to the much greater velocities observed along the $z$-axis indicating there is a region of strong acceleration in the convergence zone due to the axial pressure gradient. Using the same methodology as the experiments to determine an average velocity, the jet tip was found to move with a velocity of $4 \times 10^{4} \mathrm{~m} / \mathrm{s}$; similar to that measured in experiments. However, the instantaneous fluid velocities are a factor of $\sim 4$ times greater, at $\sim 1.5 \times 10^{5} \mathrm{~m} / \mathrm{s}$ in the jet flow region (Fig. 5), implying that the Mach number of these jets may be higher than previously estimated. The low experimental estimate of fluid velocity may be due to the use of interferometry to track the position of the jet tip. This method is constrained by the lower areal electron density limit of $\sim 10^{16} \mathrm{~cm}^{-2}$; while the assumption of an axially constant ionization level may not be appropriate due to radiative cooling. The temperature of the jet above the convergence region is $\sim 6 \mathrm{eV}$, which again agrees well with the experimental estimate. Using the fluid velocity above, the simulated jet Mach number is $>20$. As can be seen in Fig. 5b, the jet is cooler than the surrounding matter, which is $>10 \mathrm{eV}$. This supports the radiative cooling expected in these jets by Ampleford et al. (2009).

It is typically assumed experimentally that the jets from the x-pinch are hydrodynamic, and the simulations in general support this. The magnetic field map shown in Fig. $6 \mathrm{~b}$ shows that while the magnetic field is high in the jet formation region, this field strength rapidly decays with increasing distance from the cross point. There is little to no field left in the jet by the time is has propagated beyond the anode plate.

The fact that results from these simulations match those obtained in experiments increases the level of confidence in the ability to institute simulations to design scalable astrophysically relevant jet/target interaction experiments.

\section{Conclusions}

It has been demonstrated that highly supersonic, freely propagating plasma jets can be produced in low current x-pinch experiments. Quantitative two frame interferometry has been used to image jets above the anode of an x-pinch, and axial areal electron density profiles relayed a jet velocity of $3.3 \pm 0.6 \times 10^{4} \mathrm{~m} / \mathrm{s}$. A comparison of radial lineouts yielded a sound speed of $5.5 \pm 2.6 \times 10^{3} \mathrm{~m} / \mathrm{s}$, which inferred an electron temperature of $\sim 7 \mathrm{eV}$ (assuming a $Z$ of $\sim 5$ ). This gives an average Mach number of $\sim 6$, demonstrating that the jets discussed in this paper show a potential scalability to astrophysics. Estimates of the mean free path suggest a fluid description is applicable, and values of $M_{\mathrm{a}}=6$ and $\chi \sim<1$, are in the appropriate regimes. Threedimensional resistive MHD simulations demonstrated good agreement with the experimental data, and provide a basis to design future jet-target interaction experiments.

Acknowledgements The authors would like to thank Dr. David Ampleford (Sandia National Laboratory) and Dr. Simon Bland (Imperial College) for useful discussions. Work is supported by the DoE Junior Faculty Grant DE-FE02-05ER54842 and the joint DoE/NNSA HEDLP Program Grant DE-SC-0001063.

Open Access This article is distributed under the terms of the Creative Commons Attribution Noncommercial License which permits any noncommercial use, distribution, and reproduction in any medium, provided the original author(s) and source are credited.

\section{References}

Ampleford, D.J., Lebedev, S.V., Bland, S.N., Bott, S.C., Chittenden, J.P., Jennings, C.A., et al.: Phys. Plasmas 14, 102704 (2007)

Ampleford, D.J., Lebedev, S.V., Ciardi, A., et al.: Phys. Rev. Lett. 100, 035001 (2008)

Ampleford, D.J., Lebedev, S.V., Ciardi, A., Bland, S.N., Hall, G.N., Bott, S.C., Suzuki-Vidal, F., Palmer, J.B.A., Jennings, C.A., Chittenden, J.P.: AIP Conf. Proc. 1088, 83 (2009)

Beg, F.N., Ciardi, A., Ross, I., Zhu, Y., Dangor, A.E., Krushelnick, K.: IEEE Trans. Plasma Sci. 34, 2325 (2006) 
Beg, F.N., Zhang, T., Fedin, D., Beagen, B., Chua, E., Lee, J.Y., Rawat, R.S., Lee, P.: Phys. Scr. 76, 134 (2007)

Benattar, R., Popovics, C., Sigel, R.: Rev. Sci. Instrum. 50, 1583 (1979)

Chittenden, J.P., Jennings, C.A.: Phys. Rev. Lett. 101, 055005 (2008)

Chittenden, J.P., Lebedev, S.V., Jennings, C.A., Bland, S.N., Ciardi, A.: Plasma Phys. Control. Fusion 46, B457 (2004)

Haas, D., Bott, S.C., Vikhrev, V., Eshaq, Y., Ueda, U., Zhang, T., Baranova, E., Krasheninnikov, S.I., Beg, F.N.: Plasma Phys. Control. Fusion 49, 1151 (2007)

Hipp, M., Woisetschleager, J., Reiterer, P., Neger, T.: Measurement 36, 53 (2004)

Lebedev, S.V., Chittenden, J.P., Beg, F.N., Bland, S.N., Ciardi, A., Ampleford, D., Hughes, S., Haines, M.G., Frank, A., Blackman, E.G., Gardiner, T.: Astrophys. J. 564, 113 (2002)

Lebedev, S.V., Ampleford, D., Ciardi, A., Bland, S.N., Chittenden, J.P., Haines, M.G., Frank, A., Blackman, E.G., Cunningham, A.: Astrophys. J. 616, 988 (2004)

Lebedev, S.V., Ciardi, A., Ampleford, D.J., Bland, S.N., Bott, S.C., Chittenden, J.P., Hall, G.N., Rapley, J., Jennings, C.A., et al.: Mon. Not. R. Astron. Soc. 361, 97 (2005)

Mitchell, I.H., Aliaga-Rossel, R., Savedra, R., Chuaqui, H., Favre, M., Wyndham, E.S.: Phys. Plasmas 7, 5140 (2000)
Pikuz, S.A., Shelkovenko, T.A., Romanova, V.M., Hammer, D.A., Faenov, A.Ya., Dyakin, V.A., Pikuz, T.A.: Rev. Sci. Instrum. 68, 740 (1997)

Pikuz, S.A., Sinars, D.B., Shelkovenko, T.A., Chandler, K.M., Hammer, D.A., Ivanenkov, G.V., Stepniewski, W., Skobelev, I.Yu.: Phys. Rev. Lett. 89, 035003 (2002)

Ryutov, D.D., Drake, R.P., Kane, J., Liang, E., Remington, B.A., Wood-Vasey, W.M.: Astrophys. J. 518, 821 (1999)

Ryutov, D.D., Drake, R.P., Remington, B.A.: Astrophys. J. Suppl. Ser. 127, 465 (2000)

Salzmann, D.: Atomic Physics in Hot Plasmas, p. 140. Oxford University Press, New York (1998)

Shelkovenko, T.A., Sinars, D.B., Pikuz, S.A., Hammer, D.A.: Phys. Plasmas 8, 1305 (2001a)

Shelkovenko, T.A., Sinars, D.B., Pikuz, S.A., Chandler, K.M., Hammer, D.A.: Rev. Sci. Instrum. 72, 667 (2001b)

Shelkovenko, T.A., Pikuz, S.A., Sinars, D.B., Chandler, K.M., Hammer, D.A.: Phys. Plasmas 9, 2165 (2002)

Suzuki-Vidal, F., Lebedev, S.V., Ciardi, A., Bland, S.N., Chittenden, J.P., et al.: Astrophys. Space Sci. 322, 19 (2009)

Zakharov, S.M., Ivanenkov, G.V., Kolomenskii, A.A., Pikuz, S.A., Samokhin, A.I., Ulshmid, I.: Sov. Tech. Phys. Lett. 8, 456 (1982) 